\title{
Some Factors Affected on Structure, Mechanical of Ni Bulk
}

\author{
Nguyen Trong Dung*, Nguyen Chinh Cuong \\ Faculty of Physics, Hanoi National University of Education, Hanoi, Vietnam \\ Email: *dungntsphn@gmail.com
}

How to cite this paper: Dung, N.T. and Cuong, N.C. (2018) Some Factors Affected on Structure, Mechanical of Ni Bulk. Advances in Materials Physics and Chemistry, 8, 177-192.

https://doi.org/10.4236/ampc.2018.84012

Received: December 21, 2017

Accepted: April 27, 2018

Published: April 30, 2018

Copyright $\odot 2018$ by authors and Scientific Research Publishing Inc. This work is licensed under the Creative Commons Attribution International License (CC BY 4.0).

http://creativecommons.org/licenses/by/4.0/

\begin{abstract}
The article examines the effect of atomic number, temperature and tempering time on microstructure and mechanical of Ni bulk by molecular dynamics simulation and deformation z-axis. Samples $\mathrm{Ni}$ with $N=4000,5324,6912$, and 8788 atoms at $300 \mathrm{~K}, 6912$ atoms at $T=1100,900,700,500,300 \mathrm{~K}$ and 6912 atoms at $900 \mathrm{~K}$ after different annealing time. The samples were incubated with the same heating rate $\frac{\Delta T}{\Delta t}=4 \times 10^{12} \mathrm{~K} / \mathrm{s}$. Combined with common neighborhood analysis method shown in sample is always existing four types structure: FCC, HCP, BCC, and Amor. In particular, structural units FCC, HCP and Amor always prevail and BCC are very small and appear only at 300, $500 \mathrm{~K}$ with 6912 atoms. When increasing atomic number, lowering temperature or increasing tempering time will facilitate crystallization process leading to increased FCC and HCP units number. The increasing FCC, HCP units number and additional appearance BCC structure led to change microstructure and mechanical of material: When increasing atom, lowering temperature and increasing incubation time lead to an increase in density of atoms that increase mechanical properties of the material.
\end{abstract}

\section{Keywords}

Atoms Number, Temperature, Microstructure, Mechanical, Ni Bulk

\section{Introduction}

Nowadays, materials Ni bulk are always used in industries: Microelectronics [1] [2] [3] [4], photocatalyst [5] [6], photovoltaics [7], and solar energy [8]. In that, they have great influence on microstructure, surface morphology and crystallization state on microstructure, mechanical. To research and manufacture, materials have many methods: experimental [9]-[13] and theoretical [14] [15] [16]. The results do not always give the desired results. Phase transitions, heteroge- 
neous dynamics, surface shape, size, and crystallization lead to changes structures: BCC, FCC, HCP, [17]-[24]. Cause when size material limited led to appearance quantum effects (Size effects, surface effects), materials appear much different natures [25] [26] [27] [28].

To get a better understanding of the factors that influence on microstructure and mechanical of material. Molecular dynamics simulation is considered an effective tool for empirical research to study the microstructure of metals [29]-[38], deformation of z-axis to determine mechanical properties of metal have structured $\mathrm{FCC}(\mathrm{Al}, \mathrm{Cu}$ và $\mathrm{Ni})$ and $\mathrm{BCC}(\mathrm{Fe}, \mathrm{Cr}, \mathrm{W})$. Macmillan, Kelley [39] and Parrinello, Rahman found [40] that: With Ni bulk, deformation of material depends greatly on the direction of strain and the intensity of applied pressure. Komandari et al. [41] [42], Park et al. [43] [44] stretched nanowires in different directions and $\mathrm{Wu}$ [45] [46], Golovnev [47] studied the mechanical properties of $\mathrm{Cu}$ nanowires and determined relationship between temperatures. Dimensions are always proportional to deformation, and Lin Yuan and colleagues [48] examined monoclinic mechanics at different temperatures. The results show, we cannot predict the deformation of materials at high temperatures and high heating rates [49] [50] [51] [52]. Therefore, study a number of factors affecting the microstructure, and mechanics of nano-sized nanomaterials, will contribute to the fabrication of new materials [53]. In this paper, we focus on the influence of factors such as atomic number, temperature, microstructure and mechanical properties and termination; and the relationship among size, stress and number of structural units FCC, HCP, BCC, and Amor.

\section{Research Methodology}

$\mathrm{Ni}$ samples with $N=4000,5324,6912$ và 8778 atoms (respectively $\mathrm{Ni}_{4000}, \mathrm{Ni}_{5324}$, $\mathrm{Ni}_{6912}, \mathrm{Ni}_{8788}$ ) is placed in cubic with FCC structure at $0 \mathrm{~K}$ temperature and interruption radius $3.0 \AA$. After that increase temperature from $0 \mathrm{~K}$ up $2000 \mathrm{~K}$ and lower temperature from $2000 \mathrm{~K}$ down $1100,900,700,500$ and $300 \mathrm{~K}$ with heating rate is $4 \times 10^{12} \mathrm{~K} / \mathrm{s}$ and process of thermal annealing with $0.0,6 \times 10^{4}, 12 \times$ $10^{4}, 20 \times 10^{4}$ moving step, displacement time is $2 \mathrm{fs}$ (corresponding with $0.0,120$, $240,400 \mathrm{fs}$ ) break initial state to study microstructure in new states. In particular, the process of increasing temperature (lower temperature) the follows Nosé-Hoover relation [54] [55] [56]. To perform this process, we used molecular dynamics (MD) simulations with interactive embedding Sutton-Chen (1) and periodic boundary conditions [57]-[62].

$$
E_{\text {tot }}=\varepsilon \sum_{i}\left[\frac{1}{2} \sum_{j \neq i} \Phi\left(r_{i j}\right)-C \sqrt{\rho_{i}}\right] ; \Phi\left(r_{i j}\right)=\left(\frac{a_{i j}}{r_{i j}}\right)^{n}, \quad \rho_{i}=\sum_{j \neq i}\left(\frac{a_{i j}}{r_{i j}}\right)^{m}
$$

With: $r_{i j}$ is distance between two atoms $i, j ; a$ is parameter with dimension of length; $\rho_{i}$ is atomic density i; $E_{\text {tot }}$ is total energy of the system; $\Phi\left(r_{i j}\right)$ is energy between two atoms $i, j ; F\left(\rho_{i}\right)$ is interaction force on atom $i ; r_{c}$ is radius disconnect, $\varepsilon$ is energy; $C, m, n, N$ is constant. With $\varepsilon=7.3767 \mathrm{meV}, C=84.745, n=10$, 
$m=5$ và $\mathrm{a}=3.52 \AA$ selected for accurate results on microstructure of materials at different temperatures.

Previously, interactive embedding Sutton-Chen has been used extensively to study phase transition in metals [63] [64] [65] [66] [67]. In addition, study microstructure of materials we use Common Neighbor Analysis methods (CNA) [68] [69] [70]. To study mechanical properties of $\mathrm{Ni}$, we used $\mathrm{z}$-axis deformation method (2) to determine the relationship between stress and strain.

$$
\begin{gathered}
\varepsilon_{z z}=\frac{l_{z z}(t)-l_{z z}(0)}{l_{z z}(0)}=\alpha \sigma_{z z} ; \alpha=\frac{1}{E} \text { and } \sigma_{z z}=\sigma_{z z}-\mu\left(\sigma_{x x}+\sigma_{y y}\right) ; \\
G=\frac{E}{2(1+\mu)} \\
\sigma_{\alpha \beta}=\frac{1}{N} \sum_{i=1}^{N} \frac{1}{V_{i}}\left[m_{i} v_{\alpha}^{i} v_{\beta}^{i}+\frac{1}{2} \sum_{i \neq j} F_{i j} \frac{r_{i j, \alpha} r_{i j, \beta}}{r_{i j}}\right], \mu=0.31
\end{gathered}
$$

Here, $m_{i}$ is mass of atom $i, v_{\alpha}^{i}$ is velocity of atom $i$ along axis $\alpha$ and $F_{i j}$ is interaction force between atom i and atom $j, r_{i j}$ is distance between atom $i, j$, and $r_{i j, \alpha}$ is vector in $\alpha$ axis from atom i to atom $\mathrm{j}, V_{i}$ is volume of atom $i$. Where $E$ is stress, $G$ is slipping and $\mu$ is deformation coefficient, $\alpha$ is factor deformation, $\boldsymbol{\varepsilon}_{z z}$ is deformation z-axis; $\sigma_{x x} \sigma_{y p} \sigma_{z z}$ is stress according to $x, y, z$, and $\sigma_{\alpha \beta}$ is stress model. Deformation is determined by the work [71] [72].

\section{Results and Discussion}

\subsection{The Influence of the Atomic Number}

The microstructure of $\mathrm{Ni}_{4000}, \mathrm{Ni}_{5324}, \mathrm{Ni}_{6912}, \mathrm{Ni}_{8788}$ at temperature $300 \mathrm{~K}$, determined by radial distribution function (RDF), resulting in Figure 1.

The $\mathrm{Ni}_{4000}$ has the first peak of RDF prevail with valuable is $2.45 \AA$ that shows sample Ni bulk do not far order exist that always exist near order (Figure 1(a)) and have average coordination number is 12 (Figure 1(b)). When increasing atoms number then first peak height of RDF decreased from $7.35\left(\mathrm{Ni}_{4000}\right)$ down $5.76\left(\mathrm{Ni}_{6912}\right)$ and increased $7.61\left(\mathrm{Ni}_{8788}\right)$ leading to increased atomic density. The first peak of RDF increases and decreases do not follow specific rules. The minimum value at $\mathrm{Ni}_{6912}$, due to in material has microstructural units existence, results show in the second peak of RDF. The second position peak varies of RDF from 3.45 to $3.55 \AA$ reaching and a maximum value at $3.55 \AA$. The first peak height RDF Ni bulk sample smaller nanoparticles, second peak position Ni bulk larger nanoparticles. These results are entirely consistent with $\mathrm{Ni}$ nanoparticles [73]. Confirm this, we use simulation method, resulting in Figure 2 and Table 1

The results show that $\mathrm{Ni}_{4000}$ at $300 \mathrm{~K}$ has three types of structures: FCC, HCP, Amor (Figure 2(e), Figure 2(g), Figure 2(i)). When increasing $N$ from 4000 to 5324, 6912 and 8788 atoms then number units of FCC, HCP increased, Amor decrease (Figures 2 (a)-(c)) and Table 1 . However, with $\mathrm{Ni}_{6912}$ sample shows has four types structures of FCC, HCP, BCC, Amor (Figure 2(e), Figure 2(g), Figure 2(h), Figure 2(i)). When increases atoms number lead to a density of atoms 
increases, increasing units number of FCC, HCP, BCC and decrease units number Amor in the sample. The main cause leading to the appearance of number units structural of BCC is due to the size effect. The appearance of structural

Table 1. Number of atoms has structural units at $300 \mathrm{~K}$.

\begin{tabular}{ccccc}
\hline Samp (atom) & 4000 & 5324 & 6912 & 8788 \\
\hline FCC & 2044 & 3154 & 4017 & 4886 \\
HCP & 1943 & 1816 & 2025 & 3887 \\
BCC & 0 & 0 & 14 & 0 \\
Amor & 13 & 354 & 8 & 15 \\
\hline
\end{tabular}

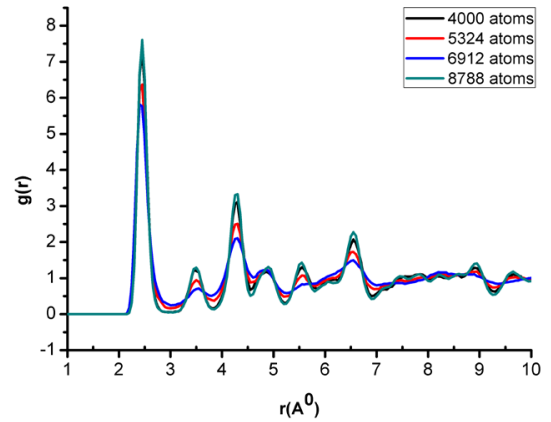

(a)

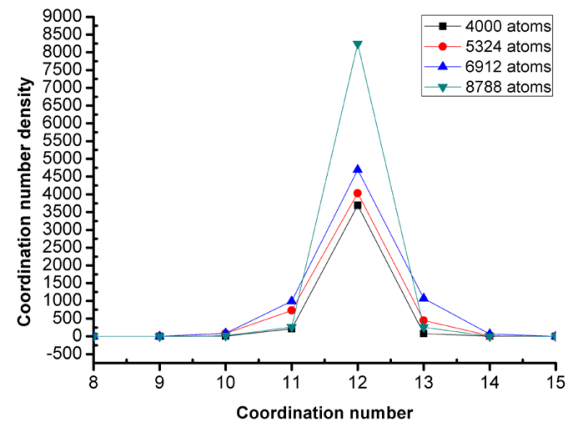

(b)

Figure 1. Radial distribution function (a); average coordination number (b) with different atomic numbers at temperature $300 \mathrm{~K}$.

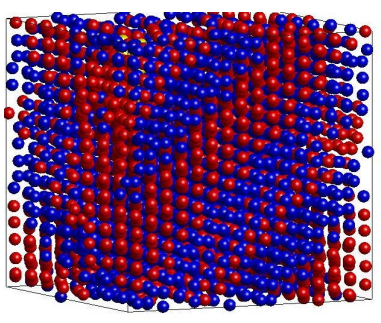

(a)

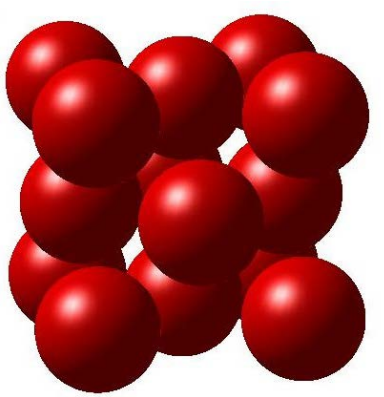

(e)

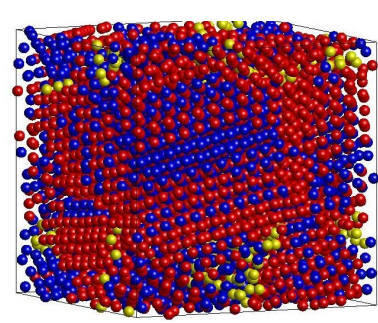

(b)

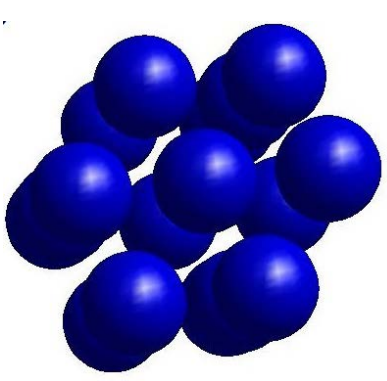

(f)

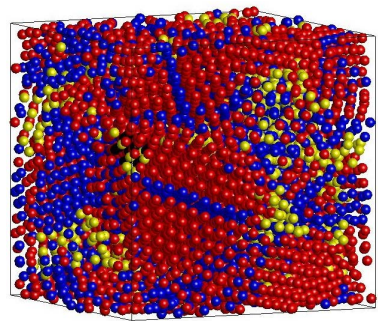

(c)

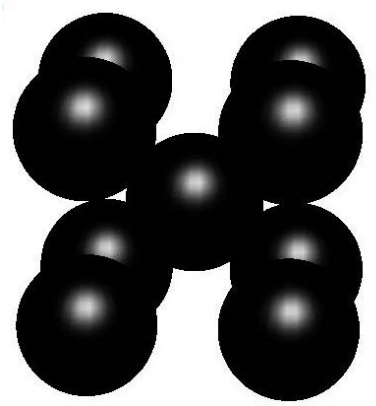

(g)

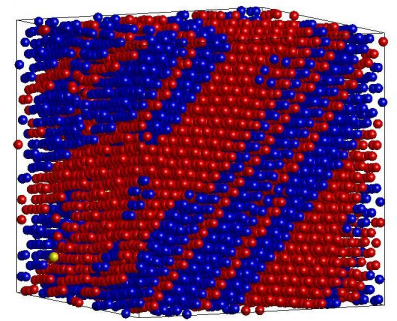

(d)

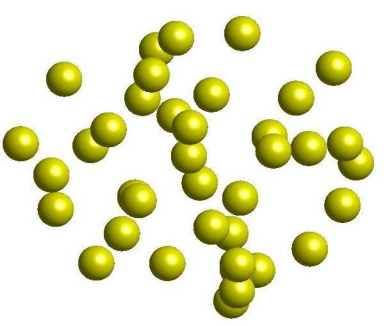

(h)

FCC Structure

HCP Structure

Amor Structure

Figure 2. New state of sample 4000 atoms (a); 5324 atoms (b); 6912 atoms (c); 8788 atoms (d) at temperature $300 \mathrm{~K}$ and structural shape in the sample (e); (g); (h); (i). 
units FCC, HCP, Amor consistent with the results [74] [75] [76] [77], unit number of BCC structure fit with Brewer [78]. With the emergence of BCC structure units, number at sample $\mathrm{Ni}_{6912}$ is astonished by scientists. However, these results should be checked and assessed for accuracy by results of Centrosymmetry Parameters [79] [80], Bond Angle Analysis [81], Bond Order [82] and another method to solve this problem. To study the influence of atomic number up mechanical properties of sample Ni bulk we used z-axis distortion method to determine the relationship between stress and strain of sample. The results are shown in Figure 3 and Table 2.

The results show, with $\mathrm{Ni}_{4000}$ sample has stress value of $E=173.04 \mathrm{GPa}$. When $\mathrm{N}$ increases from 4000 to 5324, 6912, 8788 atoms then $\mathrm{E}$ increases from 173.04 to 194.76 GPa and decreases to $189.83 \mathrm{GPa}$ and reaches a maximum value of 194.76

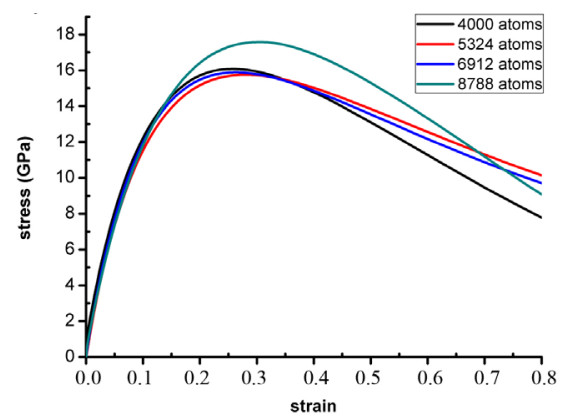

(a)

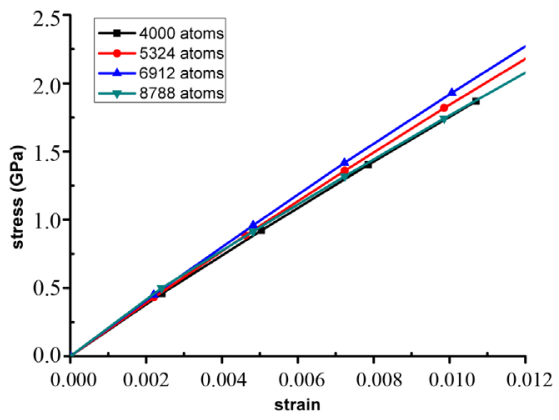

(b)

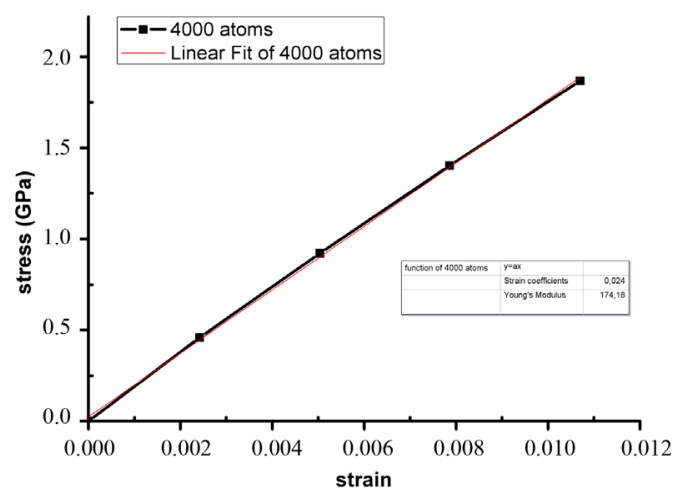

(c)

Figure 3. Relationship between deformation and stress of samples with different atomic numbers.

Table 2. Characteristics mechanical properties of Ni bulk samples at temperature $300 \mathrm{~K}$.

\begin{tabular}{ccccc}
\hline sample (atoms) & $\begin{array}{c}\text { Deformation } \\
\text { coefficient }\end{array}$ & $\begin{array}{c}\text { Stress Young } \\
\text { module E (GPa) }\end{array}$ & $\begin{array}{c}\text { Slipping module } \\
\text { G (GPa) }\end{array}$ & $\begin{array}{c}\text { Experimental results } \\
\text { at 20 } 0^{\circ} \mathrm{C} \mathrm{E} \mathrm{(GPa)}\end{array}$ \\
\hline 4000 & 0.9454 & 173.04 & 66.04 & $200[83]$ \\
5324 & 0.0069 & 181.07 & 69.11 & \\
6912 & 0.03237 & 194.76 & 74.33 & \\
8788 & 0.2004 & 189.83 & 72.45 & \\
\hline
\end{tabular}


$\mathrm{GPa}$ at $\mathrm{Ni}_{6912}$ (Figure $3(\mathrm{~b})$ ). This result is consistent with influence atoms number on microstructure and number structural units. With $\mathrm{Ni}_{6912}$ structure appears BCC and increase structure number FCC, HCP, BCC is greatest. That raises the question: Is there a link between atoms number, structural units number and stress value of the material. To answer this question, previous studies have examined the effect of porosity on mechanical properties of materials by equation Voigt [84], Spriggs [85] and correlation function Hashin-Strikman [84]. Besides, under influence of external forces leading to change of shape when the structural units number in crystal lattice changes: Ito and est [86] assume that size of empty hole increases when deformation increases lead to stress decreases. The results were consistent with experimental results, at $293 \mathrm{~K}$ with $E=$ $200 \mathrm{Gpa}$ [56], Slipping $G=79.6 \mathrm{GPa}$, deformation coefficient $\alpha=0.31$. This shows that influence of atoms number on microstructure leads to appearance BCC structure at Ni6912 to increase stress $E$.

\subsection{Effect of Temperature}

The microstructural, mechanical properties of $\mathrm{Ni}_{6912}$ bulk at $1100,900,700,500$ and $300 \mathrm{~K}$ are shown in Figure 4.

The results show that at $1100 \mathrm{~K}$ on sample there existed only 13 atoms has HCP structure (Figure 4(a1)). The first peak of radial distribution function $g(\mathrm{r})$ is $3.4 \AA$ (Figure $4(\mathrm{a} 2)$ ) and stress $E=0$ and slope $G=0$ (Figure 4(a3)). When temperature lowered 900, 700, 500 and $300 \mathrm{~K}$ then structure units number FCC, HCP and BCC increased sharply lead to Amor structure units number decrease resulting (Figures 4(b1)-4(e1)), $g(\mathrm{r})$ increased sharply from $3.4 \AA$ up $5.82 \AA$ (Figures 4(b2)-4(e2)) and $E, G$ increased from 0.0 up $194.76 \mathrm{GPa}, 74.33 \mathrm{Gpa}$ (Figures 4(b3)-4(e3)). However, phase transition from liquid to amorphous states lead to structure units number of structural, $g(\mathrm{r}), E$ and $G$ increased. The obtained results show that at $900 \mathrm{~K}$ is transition temperature $\left(T_{\mathrm{m}}\right)$ of $\mathrm{Ni}_{6912}$ bulk. This result is consistent with $\mathrm{Ni}_{4000}$ bulk has crystalline temperature is $800 \mathrm{~K}$ [32], $\mathrm{Ni}_{5324}$ nanoparticles have $T_{\mathrm{m}}=800 \mathrm{~K}$ [73] and when $N$ increases then $T_{\mathrm{m}}$ increasing [30]. In addition, phase transition temperature depends not only on number of atoms but also on shape and size [35] such as: Wen et al. assume that melting temperature $T_{\mathrm{m}}$ of $\mathrm{Ni}$ nanowires is inversely proportional with size $D$ [31] and Trong Dung Nguyen assumes that for Ni nanoparticles then $D$ is proportional with $N^{-1 / 3}, E$ is inverse with $N$ and confirm that transition temperature of sample is not applicable to nanoparticles [73]. The other side, when temperature decreases then $E, G$ increase [87]-[91] as by deformation of z-axis, $E=171$ GPa [92]. This confirms when temperature decreases then $E$ increases and determination at $900 \mathrm{~K}$ is phase transition temperature of $\mathrm{Ni}_{6912}$ bulk.

\subsection{The Effect of Crystallization}

The energy, size, number of structural units, radial distribution function, $E$ and $G$ of $\mathrm{Ni}_{6912}$ bulk after incubation time are shown in Table 3, Figure 5. 


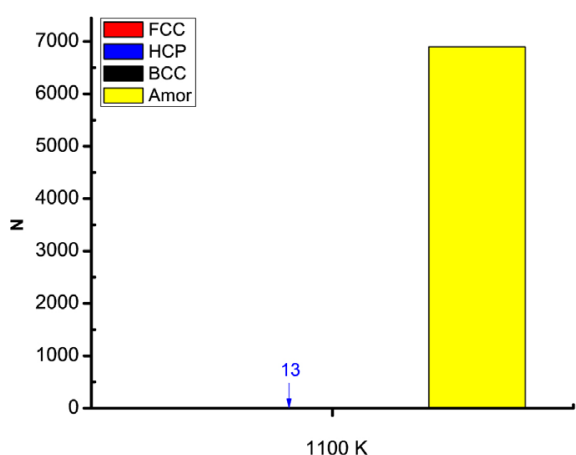

(a1)

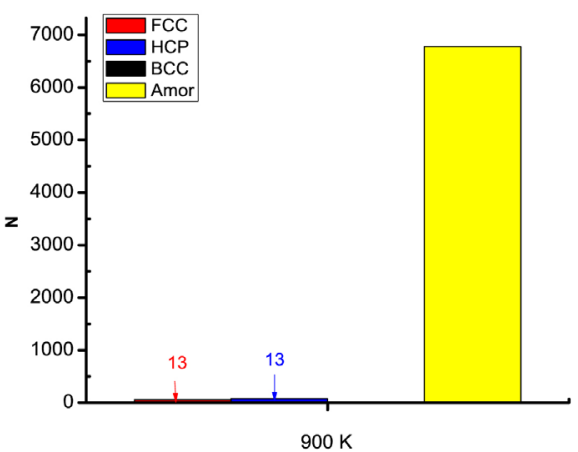

(b1)

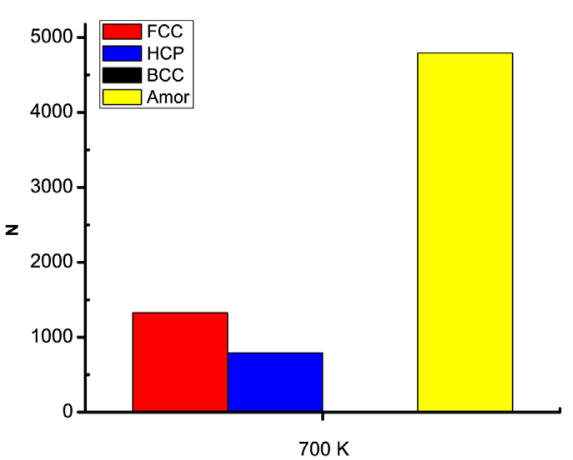

(c1)

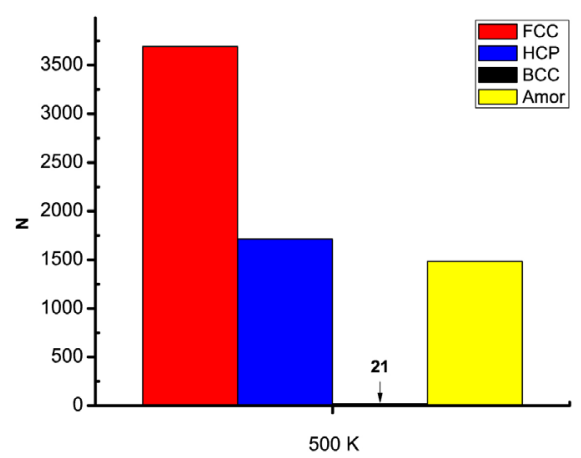

(d1)

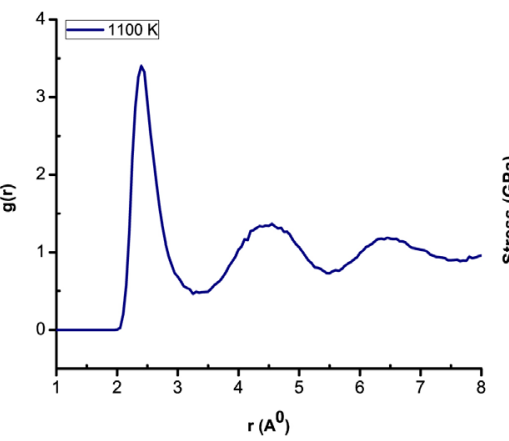

(a2)

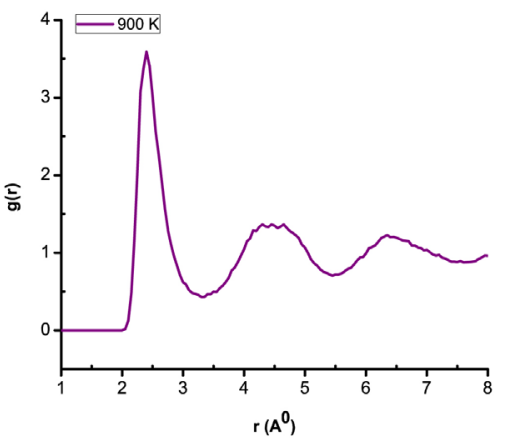

(b2)

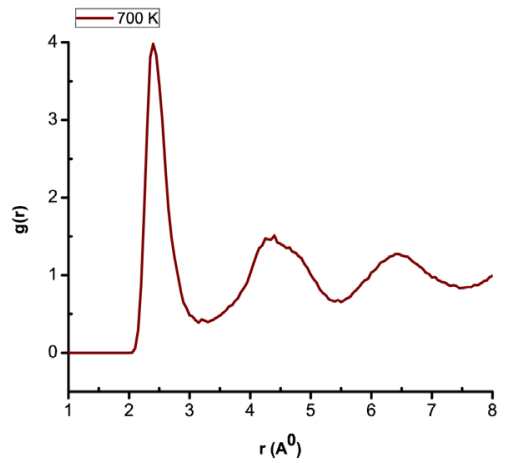

(c2)

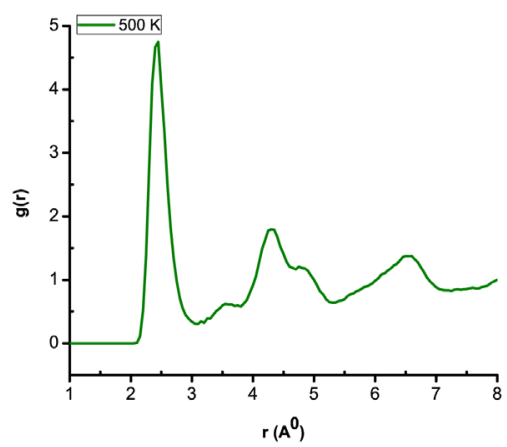

(d2)

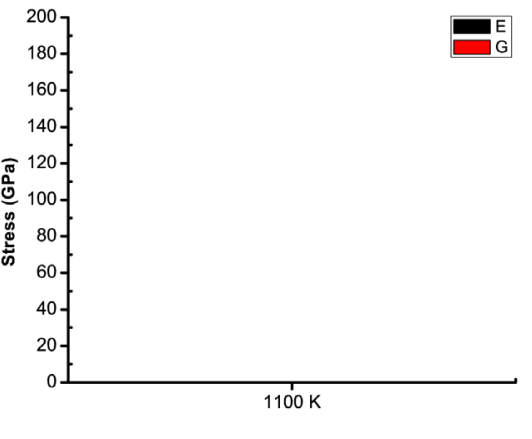

(a3)

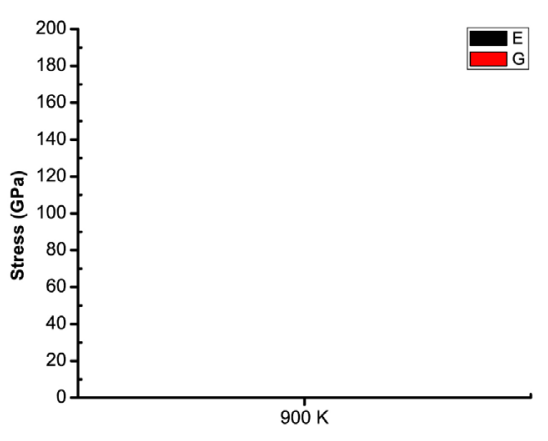

(b3)

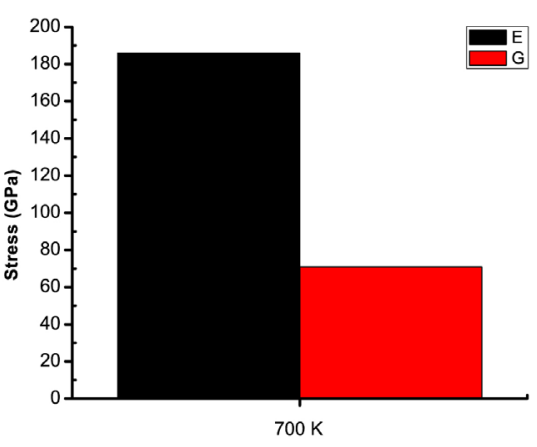

(c3)

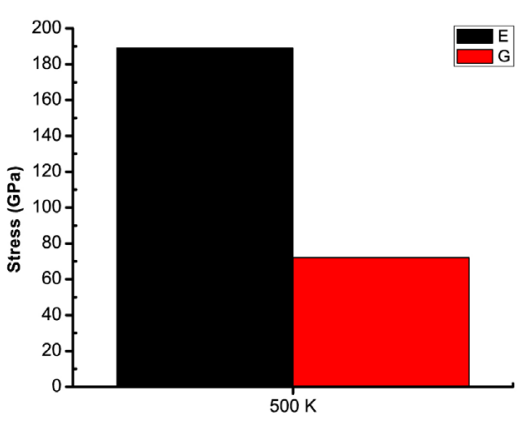

(d3) 


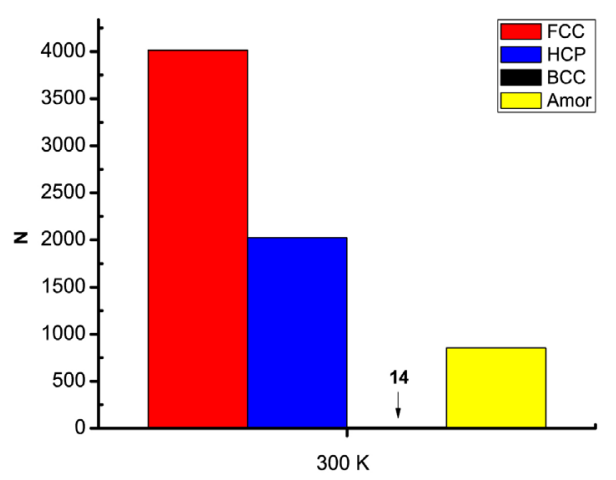

(e1)

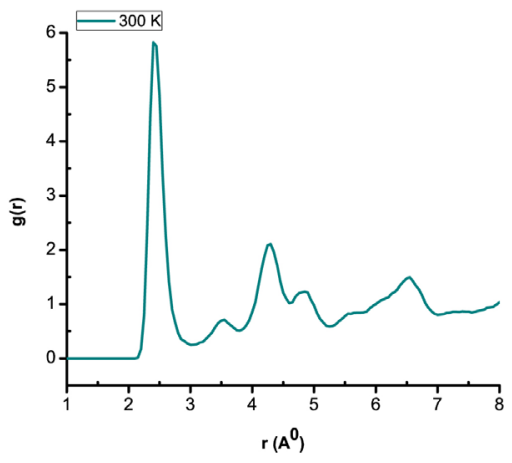

(e2)

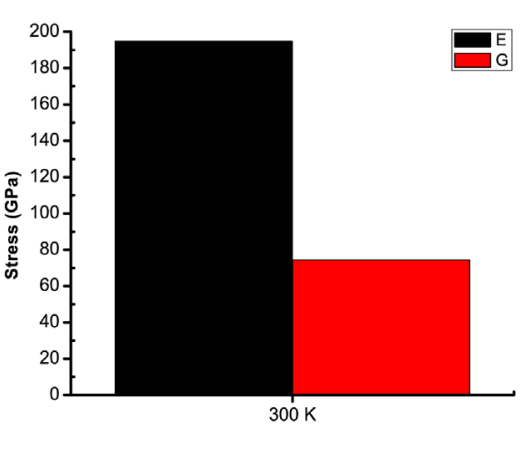

(e3)

Figure 4. The number of the structural units, microstructure and mechanical of $\mathrm{Ni}_{6912}$ bulk at different temperatures.

Table 3. Energy, size of $\mathrm{Ni}_{6912}$ bulk after different incubation time.

\begin{tabular}{ccccc}
\hline Tempering time $(\mathrm{fs})$ & 0.0 & 120 & 240 & 400 \\
\hline${\text { Energy E } \mathrm{E}_{\text {tot }}(\mathrm{eV})}_{\text {Size D }(\mathrm{nm})}^{-1097.37}$ & -1097.22 & -1099.69 & -1099.49 \\
\hline
\end{tabular}

The results show that at 0.0 fs then energy of sample is $-1097.37 \mathrm{eV}$, size is $8.209 \mathrm{~nm}$, structural units number are 56 FCC, $75 \mathrm{HCP}, g(\mathrm{r})=3.59 \AA$, $E=G=0$ $\mathrm{GPa}$. When increases the heating time up 120, 240, $400 \mathrm{fs}$ then energy, size decreases leading structural units number FCC, HCP increases, $g(\mathrm{r})$ increases, $E$ and $G$ increase. This result is consistent with simulation results of $\mathrm{Ni}_{5324}$ nanoparticles [73]. On the other hand, when increasing tempering time then $E, G$ of sample increases. After 400 fs heating time then deformation coefficient $\alpha=$ 0.01299 and stress $E=200.31 \mathrm{GPa}, G=76.46 \mathrm{GPa}$ (Figure 5(d3)). These results simulation are consistent with results of the experiment [83]. This confirms that there is a great influence of atoms number, temperature and thermal time on microstructure and mechanical properties of Ni bulk.

\section{Conclusion}

The $\mathrm{Ni}_{4000}, \mathrm{Ni}_{5324}, \mathrm{Ni}_{6912}, \mathrm{Ni}_{8788}$ samples were prepared by molecular dynamics simulation and $\mathrm{z}$-axis compression. The results showed that when increasing atomic number (decreasing temperature and increasing incubation time) then RDF height decreases (increasing) leading to structural unit number FCC, HCP, BCC, $E, G$, and mechanical properties increase. The influence of atomic number, temperature, tempering time on microstructure and mechanical properties in the material structure exists in FCC, HCP, and BCC. Based on results shown, sample Ni bulk with a higher atomic number, lower temperature, and higher thermal incubation time than sample Ni bulk will has more balanced and stable. With samples Ni bulk has an atomic number, temperature and different heating time then structural unit number FCC and HCP changes are still dominant mainly in FCC structure while structure units number BCC is very small. When increasing $N$ 


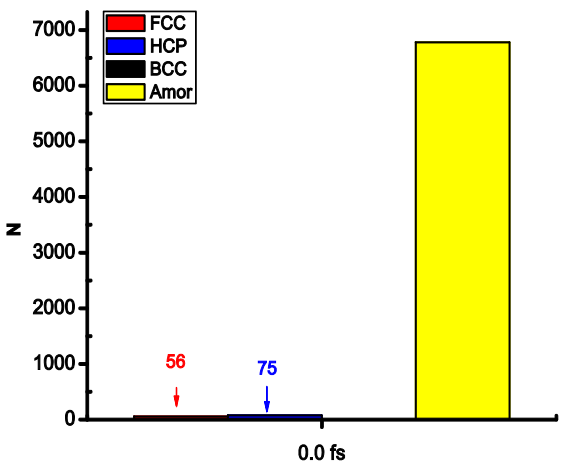

(a1)

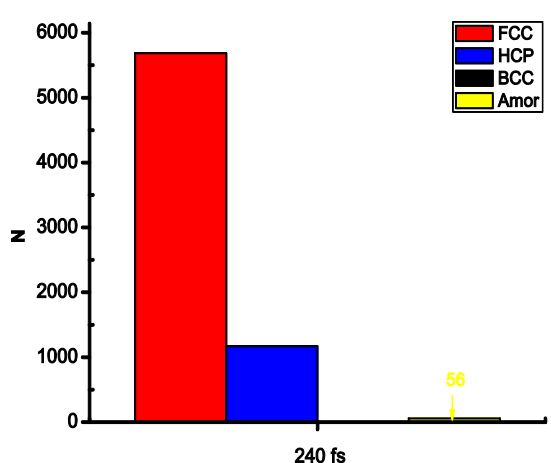

(c1)

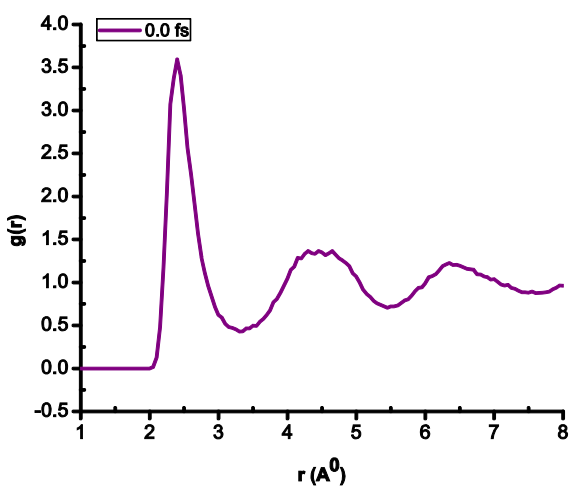

(a2)

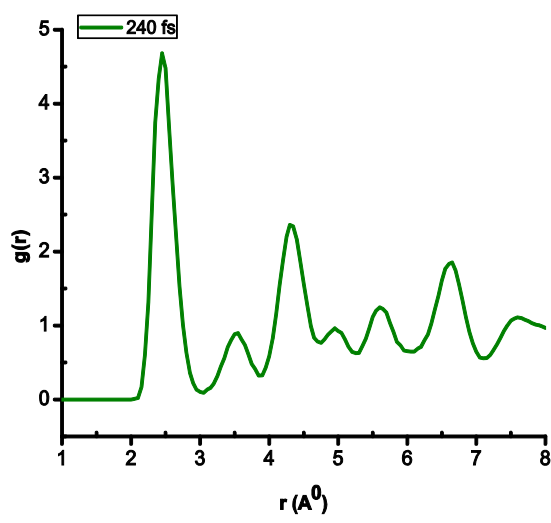

(c2)

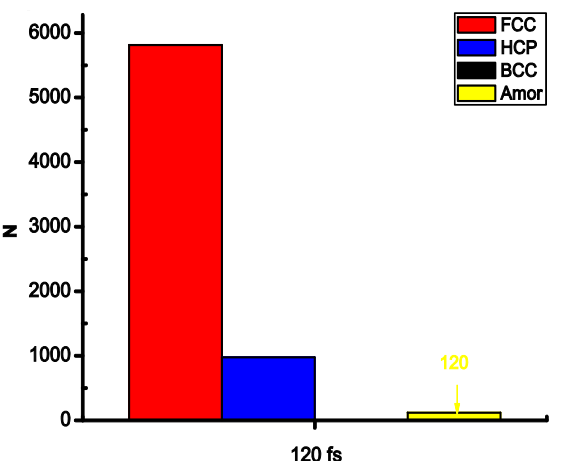

(b1)

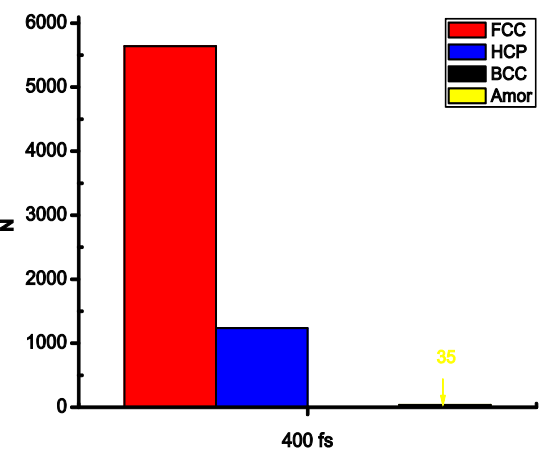

(d1)

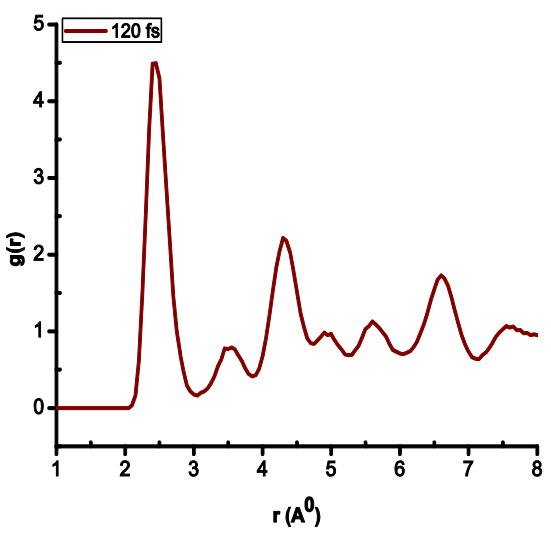

(b2)

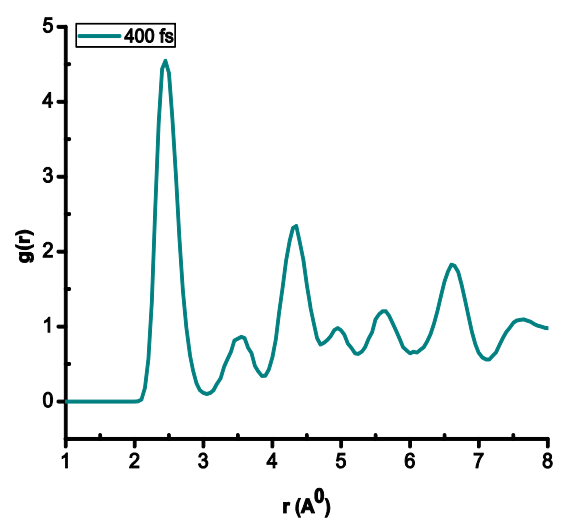

(d2) 


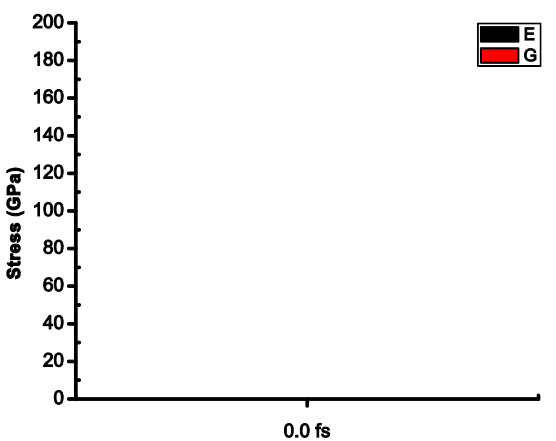

(a3)

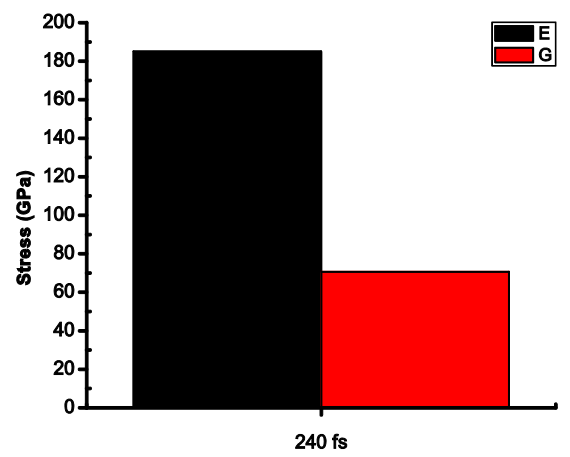

(c3)

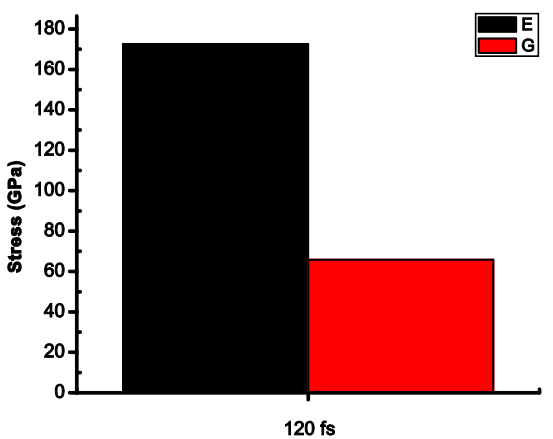

(b3)

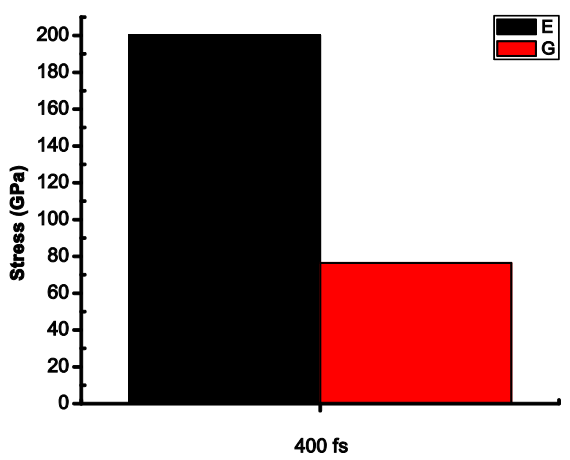

(d3)

Figure 5. Structural unit number, radial distribution function, mechanical of $\mathrm{Ni}_{6912}$ bulk after different heating time.

from 4000 to 8788 atoms then $g(r)$ decreases, structure units number FCC and HCP increase as $\alpha, E, G$ increase. At $\mathrm{Ni}_{6912}$ add structure BCC lead to $E$ reach maximum value, this suggests that addition of BCC structure increases entropy. This is an indispensable basis for the balance of Ni bulk also when decreasing temperature and increasing incubation time lead to stress increases, is due to in structure units number FCC and HCP increase. The results are based on theoretical foundations of structural units number FCC, HCP, and BCC. Amors are unclear and should be encouraged in further studies.

\section{Acknowledgements}

I thank information technology department, Faculty of Physics of Hanoi National University of Education has created all favorable conditions, help me throughout the process of calculating, simulating and finishing the content of the article.

\section{References}

[1] Karmakar, S., Kumar, S., Rinaldi, R. and Maruccio, G. (2011) Nano-Electronics and Spintronics with Nanoparticles. Journal of Physics: Conference Series, 292, Article ID: 012002. https://doi.org/10.1088/1742-6596/292/1/012002

[2] Weller, D. and Doerner, M.F. (2000) Extremely High-Density Longitudinal Magnetic Recording Media. Reviews on Advanced Materials Science, 30, 611-644. https://doi.org/10.1146/annurev.matsci.30.1.611 
[3] Sio, L.D., Placido, T., Comparelli, R., Curri, M.L., Striccoli, M., Tabiryan, N. and Bunning, T.J. (2015) Next-Generation Thermo-Plasmonic Technologies and Plasmonic Nanoparticles in Optoelectronics. Progress in Quantum Electronics, 41, 23-70. https://doi.org/10.1016/j.pquantelec.2015.03.001

[4] Muhammed, M.A.H., Döblinger, M. and Rodríguez-Fernández, J. (2015) Switching Plasmons: Gold Nanorod-Copper Chalcogenide Core-Shell Nanoparticle Clusters with Selectable Metal/Semiconductor NIR Plasmon Resonances. Journal of the American Chemical Society, 137, 11666-11677.

https://doi.org/10.1021/jacs.5b05337

[5] Wang, A., Yin, H., Ren, M., Lu, H., Xue, J. and Jiang, T. (2010) Preparation of Nickel Nanoparticles with Different Sizes and Structures and Catalytic Activity in the Hydrogenation of p-nitrophenol. New Journal of Chemistry, 34, 708-713.

https://doi.org/10.1039/b9nj00657e

[6] Morozov, Y.G., Belousova, O.V. and Kuznetsov, M.V. (2011) Preparation of Nickel Nanoparticles for Catalytic Applications. Inorganic Materials, 47, 36-40. https://doi.org/10.1134/S0020168510121027

[7] Ruan, Y., Wang, C. and Jiang, J. (2016) Nanostructured Ni Compounds as Electrode Materials towards High-Performance Electrochemical Capacitors. Journal of Materials Chemistry A, 4, 14509-14538. https://doi.org/10.1039/C6TA05104A

[8] Gaouyat, L., He, Z., Colomer, J.-F., Schryvers, D., Mirabella, F. and Deparis, O. (2015) Linking Optical Properties and Nanostructure of NiCrOx Cermet Nanocomposite for Solar Thermal Application, Springer. Nano-Structures for Optics and Photonics, 2015, 497.

[9] Schmidt, H. (2001) Nanoparticles by Chemical Synthesis, Processing to Materials and Innovative Applications. Applied Organometallic Chemistry, 15, 331-343. https://doi.org/10.1002/aoc.169

[10] Huang, K.-C. and Ehrman, S.H. (2007) Synthesis of Iron Nanoparticles via Chemical Reduction with Palladium Ion Seeds. Langmuir, 23, 1419-1426. https://doi.org/10.1021/la0618364

[11] Goia, D.V. (2004) Preparation and Formation Mechanisms of Uniform Metallic Particles in Homogeneous Solutions. Journal of Materials Chemistry, 14, 451-458. https://doi.org/10.1039/b311076a

[12] Tabrizi, N.S., Xu, Q., van der Pers, N.M., Lafont, U. and Schmidt-Ott, A. (2008) Synthesis of Mixed Metallic Nanoparticles by Spark Discharge. Journal of Nanoparticle Research, 11, 1209-1218. https://doi.org/10.1007/s11051-008-9568-8

[13] Förster, H., Wolfrum, C. and Peukert, W. (2012) Experimental Study of Metal Nanoparticle Synthesis by an Arc Evaporation/Condensation Process. Journal of Nanoparticle Research, 14, 926-932. https://doi.org/10.1007/s11051-012-0926-1

[14] Daw, M. and Baskes, M. (1984) Embedded-Atom Method: Derivation and Application to Impurities, Surfaces, and Other Defects in Metals. Physical Review B, 29, 6443-6453. https://doi.org/10.1103/PhysRevB.29.6443

[15] Ju, S.P., Lin, J.S. and Lee, W.J. (2004) A Molecular Dynamics Study of the Tensile Behaviour of Ultrathin Gold Nanowires. Nanotechnology, 15, 1221-1225. https://doi.org/10.1088/0957-4484/15/9/019

[16] Liew, K.M., He, X.Q. and Wong, C.H. (2004) On the Study of Elastic and Plastic Properties of Multi-Walled Carbon Nanotubes under Axial Tension using Molecular Dynamics Simulation. ACTA Material, 52, 2521-2527.

https://doi.org/10.1016/j.actamat.2004.01.043 
[17] Contescu, C.I. and Putyera, K. (2009) Dekker Encyclopedia of Nanoscience and Nanotechnology. 2nd Edition, CRC Press, Boca Raton.

[18] Cao, G. and Wang, Y. (2011) Nanostructures \& Nanomaterials: Synthesis, Properties, and Applications. World Scientific Series in Nanoscience and Nanotechnology, Vol. 2, World Scientific, Hackensack.

[19] Mitin, V.V., Sementsov, D.I. and Vagidov, N.Z. (2010) Quantum Mechanics for Nanostructures. Cambridge University Press, Cambridge.

https://doi.org/10.1017/CBO9780511845161

[20] Roduner, E. (2007) Nanoscopic Materials: Size-Dependent Phenomena. Royal Society of Chemistry, Cambridge.

[21] Cleri, F. and Rosato, V. (1993) Tight-Binding Potentials for Transition Metals and Alloys. Physical Review B, 48, 22-33. https://doi.org/10.1103/PhysRevB.48.22

[22] Chamati, H. and Papanicolaou, N.I. (2004) Second-Moment Interatomic Potential for Gold and Its Alication to Molecular-Dynamics Simulations. Journal of Physics. Condensed Matter, 16, 8399-8407. https://doi.org/10.1088/0953-8984/16/46/025

[23] Chamati, H. (2011) Journal of Materials Science \& Technology, 19, 42-51.

[24] Martin, T.P. (1996) Shells of Atoms. Physics Reports, 273, 199-241. https://doi.org/10.1016/0370-1573(95)00083-6

[25] Karaman, I., Sehitoglu, H., Chumlyakov, Y.I., Maier, H.J. and Kireeva, I.V. (2001) The Effect of Twinning and Slip on the Bauschinger Effect of Hadfield Steel Single Crystals. Metallurgical and Materials Transactions A, 32, 695-706.

[26] Wang, Z.L., et al. (2001) Nano-Scale Mechanics of Nanotubes, Nanowires, and Nanobelts. Advanced Engineering Materials, 3, 657-661. https://doi.org/10.1002/1527-2648(200109)3:9<657::AID-ADEM657>3.0.CO;2-0

[27] Erts, D. and Olin, H. (2003) Metallic and Semiconducting Nanowires: Properties and Architectures. Proceedings of SPIE, 5123, 248-258. https://doi.org/10.1117/12.517030

[28] Yang, Z. and Zhao, Y.-P. (2007) Size-Dependent Elastic Properties of Ni Nanofilms by Molecular Dynamics Simulation. Surface Review and Letters, 14, 661-665. https://doi.org/10.1142/S0218625X07010032

[29] Lu, J. and Szpunar, J.A. (1997) Applications of the Embedded-Atom Method to Glass Formation and Crystallization of Liquid and Glass Transition-Metal Nickel. Philosophical Magazine A, 75, 1057-1066. https://doi.org/10.1080/01418619708214010

[30] Qi, Y., Çagin, T., Johnson, W.L. and Goddard III, W.A. (2001) Melting and Crystallization in Ni Nanoclusters: The Mesoscale Regime. The Journal of Chemical Physics, 115, 385-394. https://doi.org/10.1063/1.1373664

[31] Wen, Y.-H., Zhu, Z.-Z., Zhu, R. and Shao, G.-F. (2004) Molecular Dynamics Study of the Mechanical Behavior of Nickel Nanowire: Strain Rate Effects. Physica E: Lowdimensional Systems and Nanostructures, 25, 47-54. https://doi.org/10.1016/j.physe.2004.06.048

[32] Zhang, Y., Wang, L. and Wang, W. (2007) Thermodynamic, Dynamic and Structural Relaxation in Supercooled Liquid and Glassy Ni below the Critical Temperature. Journal of Physics. Condensed Matter, 19, Article ID: 196106. https://doi.org/10.1088/0953-8984/19/19/196106

[33] Kien, P.H. (2014) Study of Structural and Phase Transition of Nickel Metal. ISRN Materials Science, 2014, Article ID: 253627. 
[34] Andriotis, A.N., Fthenakis, Z.G. and Menon, M. (2007) Correlated Variation of Melting and Curie Temperatures of Nickel Clusters. Physical Review B, 75, Article ID: 073413. https://doi.org/10.1103/PhysRevB.75.073413

[35] Lu, H.M., Li, P.Y., Cao, Z.H. and Meng, X.K. (2009) Size-, Shape-, and Dimensionality-Dependent Melting Temperatures of Nanocrystals. The Journal of Physical Chemistry C, 113, 7598-7602. https://doi.org/10.1021/jp900314q

[36] Karaman, I., Sehitoglu, H., Chumlyakov, Y.I., Maier, H.J. and Kireeva, I.V. (2001) Extrinsic Stacking Faults and Twinning in Hadfield Manganese Steel Single Crystals. Scripta Materialia, 44, 337-343. https://doi.org/10.1016/S1359-6462(00)00600-X

[37] Tadmor, E.B., Bernstein, N. and Mech, J. (2004) A First-Principles Measure for the Twinnability of FCC Metals. Journal of the Mechanics and Physics of Solids, 52, 2507-2519. https://doi.org/10.1016/j.jmps.2004.05.002

[38] Bernstein, N. and Tadmor, E.B. (2004) Tight-Binding Calculations of Stacking Energies and Twinnability in FCC Metals. Physical Review B, 69, Article ID: 094116. https://doi.org/10.1103/PhysRevB.69.094116

[39] Macmillan, N.H. and Kelly, A. (1972) The Mechanical Properties of Perfect Crystals I. The Ideal Strength. Proceedings of the Royal Society (London) A, 330, 291-308. https://doi.org/10.1098/rspa.1972.0146

[40] Parinello, M. and Rahman, A. (1981) Polymorphic Transitions in Single Crystals A New Molecular Dynamics Method. Journal of Applied Physics, 52, 7182-7190. https://doi.org/10.1063/1.328693

[41] Komandori, R. and Chadrasekaran, N. (2002) Molecular Dynamic Simulations of Uniaxial Tension at Nanoscale of Semiconductor Materials for Micro-Electro-Mechanical Systems (MEMS) Applications. Materials Science and Engineering: A, 340, 58-67. https://doi.org/10.1016/S0921-5093(02)00156-9

[42] Komandori, R. and Chadrasekaran, N. (2001) Molecular Dynamics (MD) Simulation of Uniaxial Tension of Some Single-Crystal Cubic Metals at Nanolevel. International Journal of Mechanical Sciences, 43, 2260-2337.

[43] Park, H.S., Gallb, K. and Zimmerman, J.A. (2006) Deformation of FCC Nanowires by Twinning and Slip. Journal of the Mechanics and Physics of Solids, 54, 1862-1881. https://doi.org/10.1016/j.jmps.2006.03.006

[44] Park, H.S. and Zimmerman, J.A. (2005) Modelling Inelasticity and Failure in Gold Nanowires. Physical Review B, 72, Article ID: 054106.

[45] Wu, H.A. (2006) Molecular Dynamics Study of the Mechanics of Metal Nanowires at Finite Temperature. European Journal of Mechanics-A/Solids, 25, 370-377. https://doi.org/10.1016/j.euromechsol.2005.11.008

[46] Wu, H.A. (2006) Molecular Dynamics Study on Mechanics of Metal Nanowire. Mechanics Research Communications, 33, 9-16. https://doi.org/10.1016/j.mechrescom.2005.05.012

[47] Golovnev, I.F., Golovneva, E.I. and Fomin, V.M. (2006) Molecular-Dynamic Modeling of Mechanical Properties of Free Defect Metal Nanocrystals. Computational Materials Science, 37, 336-348. https://doi.org/10.1016/j.commatsci.2005.09.005

[48] Yuan, L., Shan, D. and Guo, B. (2007) Molecular Dynamics Simulation of Tensile Deformation of Nano-Single Crystal Aluminum. Journal of Materials Processing Technology, 184, 1-5. https://doi.org/10.1016/j.jmatprotec.2006.10.042

[49] Branicio, P.S. and Rino, J.P. (2000) Large Deformation and Amorphization of Ni Nanowires under Uniaxial Strain: A Molecular Dynamics Study. Physical Review B, 
62, 16950-16955. https://doi.org/10.1103/PhysRevB.62.16950

[50] Ikeda, H., Qi, Y., Cagin, T., Samwer, K., Johnson, W.L. and Goddard, W.A. (1999) Strain Rate Induced Amorphization in Metallic Nanowires. Physical Review Letters, 82, 2900-2903. https://doi.org/10.1103/PhysRevLett.82.2900

[51] Landman, U., Luedtke, W.D., Salisbury, B.E. and Whetten, R.L. (1996) Reversible Manipulations of Room Temperature Mechanical and Quantum Transport Properties in Nanowire Junctions. Physical Review Letters, 77, 1362-1365. https://doi.org/10.1103/PhysRevLett.77.1362

[52] Walsh, P., Li, W., Kalia, R.K., Nakano, A., Vashishta, P. and Saini, S. (2001) Structural Transformation, Amorphization, and Fracture in Nanowires: A Multimillion-Atom Molecular Dynamics Study. Applied Physics Letters, 78, 3328-3330. https://doi.org/10.1063/1.1374237

[53] Tsuzuki, H., Branicio, P.S. and Rino, J.P. (2007) Structural Characterization of Deformed Crystals by Analysis of Common Atomic Neighborhood. Computer Physics Communications, 177, 518-523. https://doi.org/10.1016/j.cpc.2007.05.018

[54] Nosé, S. (1984) The Journal of Chemical Physics, 81.

[55] Hoover, W.G. (1985) Canonical Dynamics: Equilibrium Phase-Space Distributions. Physical Review A, 31, 1695-1697. https://doi.org/10.1103/PhysRevA.31.1695

[56] Evans, D.J. and Holian, B.L. (1985) The Nose-Hoover Thermostat. The Journal of Chemical Physics, 83, 4069-4074. https://doi.org/10.1063/1.449071

[57] Todd, B.D. and Lynden-Bell, R.M. (1993) Surface and Bulk Properties of Metals Modelled with Sutton-Chen Potentials. Surface Science, 281, 191-206. https://doi.org/10.1016/0039-6028(93)90868-K

[58] Sutton, A.P. and Chen, J. (1990) Long-Range Finnis-Sinclair Potentials. Philosophical Magazine Letters, 61, 139-146.

[59] Daw, M.S. and Baskes, M.I. (1983) Semiempirical, Quantum Mechanical Calculation of Hydrogen Embrittlement in Metals. Physical Review Letters, 50, 1285-1288. https://doi.org/10.1103/PhysRevLett.50.1285

[60] Daw, M.S. and Baskes, M.I. (1984) Embedded-Atom Method: Derivation and Alication to Impurities, Surfaces, and Other Defects in Metals. Physical Review B, 29, 6443-6453. https://doi.org/10.1103/PhysRevB.29.6443

[61] Çagin, T., Qi, Y., Li, H., Kimura, Y., Ikeda, H., Johnson, W.L. and Goddard III, W.A. (1999) Materials Research Society, 554, 43-48.

[62] Kimura, Y., Qi, Y., Çagin, T. and Goddard III, W.A. (1998) The Quantum Sutton-Chen Many-Body Potential for Properties of FCC Metals.

[63] Akbarzadeh, H. and Parsafar, G.A. (2009) A Molecular-Dynamics Study of Thermal and Physical Properties of Platinum Nanoclusters. Fluid Phase Equilibria, 280, 16-21. https://doi.org/10.1016/j.fluid.2009.02.018

[64] Qi, Y., Çagin, T., Kimura, Y. and Goddard III, W.A. (1999) Molecular-Dynamics Simulations of Glass Formation and Crystallization in Binary Liquid Metals: $\mathrm{Cu}-\mathrm{Ag}$ and Cu-Ni. Physical Review B, 59, 3527-3533. https://doi.org/10.1103/PhysRevB.59.3527

[65] Sankaranarayanan, S.K.R.S., Bhethanabotla, V.R. and Joseph, B. (2005) Molecular Dynamics Simulation Study of the Melting of Pd-Pt Nanoclusters. Physical Review B, 71, Article ID: 195415. https://doi.org/10.1103/PhysRevB.71.195415

[66] Fernández-Navarro, C. and Mejía-Rosales, S. (2013) Molecular Dynamics of Free and Graphite-Supported Pt-Pd Nanoparticles. Advances in Nanoparticles, 2, 
323-328. https://doi.org/10.4236/anp.2013.24044

[67] Kart, H.H., Uludogan, M., Çagin, T. and Tomak, M. (2004) Kluwer Academic Publishers, Dordrecht, Boston, London, 487-493.

[68] Sankaranarayanan, S.K.R.S., Bhethanabotla, V.R. and Joseph, B. (2005) Molecular Dynamics Simulation Study of the Melting of Pd-Pt Nanoclusters. Physical Review B, 71, Article ID: 195415. https://doi.org/10.1103/PhysRevB.71.195415

[69] Honeycutt, J.D. and Andersen, H.C. (1987) Molecular Dynamics Study of Melting and Freezing of Small Lennard-Jones Clusters. The Journal of Physical Chemistry, 91, 4950-4963. https://doi.org/10.1021/j100303a014

[70] Tsuzuki, H., Branicio, P.S. and Rino, J.P. (2007) Structural Characterization of Deformed Crystals by Analysis of Common Atomic Neighborhood. Computer Physics Communications, 177, 518-523. https://doi.org/10.1016/j.cpc.2007.05.018

[71] Berendsen, H.J.C., Postma, J.P.M. and van Gunsteren, W.F. (1984) Molecular Dynamics with Coupling to an External Bath. The Journal of Chemical Physics, 81, 3684-3690. https://doi.org/10.1063/1.448118

[72] Koh, S.J.A. and Lee, H.P. (2006) Molecular Dynamics Simulation of Size and Strain Rate Dependent Mechanical Response of FCC Metallic Nanowires. Nanotechnology, 17, 3451-3467. https://doi.org/10.1088/0957-4484/17/14/018

[73] Nguyen, T.D., Nguyen, C.C. and Tran, V.H. (2017) Molecular Dynamics Study of Microscopic Structures, Phase Transitions and Dynamic Crystallization in Ni Nanoparticles. RSC Advances, 7, 25406-25413. https://doi.org/10.1039/C6RA27841H

[74] Zharkov, S., Zhigalov, V. and Frolov, G. (1996) A Hexagonal Close-Packed Phase in Nickel Films. The Physics of Metals and Metallography, 81, 328-330.

[75] Mi, Y., Yuan, D., Liu, Y., Zhang, J. and Xiao, Y. (2005) Synthesis of Hexagonal Close-Packed Nanocrystalline Nickel by a Thermal Reduction Process. Materials Chemistry and Physics, 89, 359-361. https://doi.org/10.1016/j.matchemphys.2004.09.012

[76] Chiang, R.-T., Chiang, R.-K. and Shieu, F.-S. (2014) Emergence of Interstitial-Atom-Free HCP Nickel Phase during the Thermal Decomposition of Ni3C Nanoparticles. RSC Advances, 4, 19488-19494. https://doi.org/10.1039/c4ra01874e

[77] Tian, C.S., Qian, D., Wu, D., He, R.H., Wu, Y.Z., Tang, W.X., Yin, L.F., Shi, Y.S., Dong, G.S., Jin, X.F., Jiang, X.M., Liu, F.Q., Qian, H.J., Sun, K., Wang, L.M., Rossi, G., Qiu, Z.Q. and Shi, J. (2005) Body-Centered-Cubic Ni and Its Magnetic Properties. Physical Review Letters, 94, Article ID: 137210. https://doi.org/10.1103/PhysRevLett.94.137210

[78] Brewer, L. (1968) Bonding and Structures of Transition Metals. Science, 161, 115-122. https://doi.org/10.1126/science.161.3837.115

[79] Kelchner, C.L., Plimpton, S.J. and Hamilton, J.C. (1998) Dislocation Nucleation and Defect Structure during Surface Indentation. Physical Review B, 58, 11085-11088. https://doi.org/10.1103/PhysRevB.58.11085

[80] Li, J. (2003) AtomEye: An Efficient Atomistic Configuration Viewer. Modelling and Simulation in Materials Science and Engineering, 11, 173-177. https://doi.org/10.1088/0965-0393/11/2/305

[81] Ackland, G.J. and Jones, A.P. (2006) Applications of Local Crystal Structure Measures in Experiment and Simulation. Physical Review B, 73, Article ID: 054104. https://doi.org/10.1103/PhysRevB.73.054104

[82] Steinhardt, P.J., Nelson, D.R. and Ronchetti, M. (1983) Bond-Orientational Order in Liquids and Glasses. Physical Review B, 28, 784-805. 
https://doi.org/10.1103/PhysRevB.28.784

[83] Wikipedia Niken. https://vi.wikipedia.org/wiki/Niken

[84] Pabst, W., Gregorova, E. and Ticha, G. (2006) Elasticity of Porous Ceramics-A Critical Study of Modulus-Porosity Relations. Journal of the European Ceramic Society, 26, 1085-1097. https://doi.org/10.1016/j.jeurceramsoc.2005.01.041

[85] Pickup, R. (1997) Effect of Porosity on Young's Modulus of a Porcelain. British Ceramic Transactions, 96, 96-98.

[86] Ito, S., Taniguchi, T., Ono, M. and Uemura, K. (2012) Network and Void Structures for Glasses with a Higher Resistance to Crack Formation. Journal of Non-Crystalline Solids, 358, 3453-3458. https://doi.org/10.1016/j.jnoncrysol.2012.02.039

[87] Chang, W.J. and Fang, T.H. (2003) Influence of Temperature on Tensile and Fatigue Behavior of Nanoscale Coer using Molecular Dynamics Simulation. Journal of Physics and Chemistry of Solids, 64, 1279-1283. https://doi.org/10.1016/S0022-3697(03)00130-6

[88] Pethica, J.B., Hutchings, R. and Oliver, W.C. (1983) Hardness Measurement at Penetration Depths as Small as $20 \mathrm{~nm}$. Philosophical Magazine A, 48, 593-606. https://doi.org/10.1080/01418618308234914

[89] Selinger, R.L.B., Wang, Z.G. and Gelbart, W.M. (1991) Statistical-Thermodynamic Approach to Fracture. Physical Review A, 43, 4396-4400. https://doi.org/10.1103/PhysRevA.43.4396

[90] Lynden-Bell, R.M. (1994) Computer Simulations of Fracture at the Atomic Level. Science, 263, 1704-1705. https://doi.org/10.1126/science.263.5154.1704

[91] Lynden-Bell, R.M. (1995) A Simulation Study of Induced Disorder, Failure and Fracture of Perfect Metal Crystals under Uniaxial Tension. Journal of Physics: Condensation of Matter, 7, 4603-4624. https://doi.org/10.1088/0953-8984/7/24/003

[92] Rentsch, R. and Inasaki, I. (1995) Investigation of Surface Integrity by Molecular Dynamics Simulation. Annals of CIRP, 44, 295-298.

https://doi.org/10.1016/S0007-8506(07)62329-4 